\title{
Reinternamentos Hospitalares em Portugal na Última Década
}

\author{
Hospital Readmissions in Portugal over the Last Decade
}

Bernardo SOUSA-PINTO ${ }^{1}$, Ana Rita GOMES ${ }^{1}$, Andreia OLIVEIRA ${ }^{1}$, Carlos IVO ${ }^{1}$, Gustavo COSTA $^{1}$, João RAMOS ${ }^{1}$, Joel SILVA ${ }^{1}$, Maria Carolina CARNEIRO ${ }^{1}$, Maria João DOMINGUES ${ }^{1}$, Maria João CUNHA ${ }^{1}$, Altamiro da COSTA-PEREIRA ${ }^{1,2}$, Alberto FREITAS 1,2

Acta Med Port 2013 Nov-Dec;26(6):711-720

\section{RESUMO}

Introdução: Os reinternamentos hospitalares estão associados a um incremento das despesas com a saúde e da mortalidade intrahospitalar. Neste trabalho, pretende-se caracterizar os reinternamentos hospitalares não-planeados, ocorridos num período de 30 dias após alta, de acordo com a sua Grande Categoria Diagnóstica, contexto hospitalar e características demográficas dos utentes, bem como estimar as taxas de mortalidade associadas. Pretende-se também estudar a evolução da taxa de reinternamentos na última década (2000-2008). Procurar-se-á ainda caracterizar os reinternamentos por insuficiência cardíaca.

Material e Métodos: Procedeu-se à análise estatística da base de dados de internamentos hospitalares públicos fornecida pela Autoridade Central do Sistema de Saúde. Recorreu-se aos testes do qui-quadrado e de tendência para comparação de taxas de reinternamentos.

Resultados: Das 5514331 hospitalizações não-planeadas no período em estudo, 4,1\% corresponderam a reinternamentos hospitalares. Entre 2000 e 2008, a taxa de reinternamentos hospitalares aumentou continuamente de 3,0\% para 4,7\%. A mortalidade hospitalar foi significativamente maior entre os episódios de reinternamento $(9,5 \%)$ do que nos restantes episódios $(5,6 \%), p<0,001$. A taxa de reinternamentos foi significativamente maior em homens ( $4,5 \%$ versus $3,9 \%$ nas mulheres, $p<0,001)$ e doentes mais velhos ( $2,6 \%$ nas crianças e $5,3 \%$ nos idosos), sendo menor na região de Lisboa $(2,7 \%)$ e nos hospitais centrais $(3,0 \%, p<0,001)$. Para os episódios de insuficiência cardíaca, foi obtida uma taxa de reinternamentos de $6,7 \%$.

Discussão e Conclusão: Em termos gerais, as diferenças encontradas são similares às descritas noutros Países Ocidentais. Os episódios de reinternamento, cujas taxas têm vindo a aumentar em Portugal, estão associados a maior mortalidade intra-hospitalar.

Palavras-chave: Mortalidade Hospitalar; Portugal; Qualidade de Cuidados de Saúde; Readmissão do Doente.

\section{ABSTRACT}

Introduction: Hospital readmissions are associated with increased healthcare expenses and with higher hospital fatality rates. We aim to characterize unplanned hospital readmissions occurred within 30 days after discharge, according to its Major Diagnosis Category, hospital type and location, and patients' demographic attributes. We also intend to estimate the hospital fatality rates associated to those readmissions, as well as to study the evolution of hospital readmissions rates in the last decade (2000-2008). Moreover, we aim to characterize heart failure readmissions.

Material and Methods: We analysed a database (provided by Autoridade Central do Sistema de Saúde) containing all hospital admissions occurred in Portuguese public hospitals. In order to compare readmissions rates, we performed chi-square tests and linear-bylinear association tests.

Results: Between 2000 and 2008, there were 5514331 unplanned admissions, of which $4.1 \%$ corresponded to hospital readmissions, classified with the same Major Diagnosis Category of the first admission. Between 2000 and 2008, hospital readmissions rate increased continuously from $3.0 \%$ to $4.7 \%$. Hospital fatality rate was significantly higher among readmitted cases $(9.5$ versus $5.6 \%, p<0.001)$. Readmissions rates were also significantly higher among episodes involving older patients $(2.6 \%$ in children versus $5.3 \%$ in the elderly) and males $(4.5 \%$ versus $3.9 \%$ in females, $p<0.001)$, being lower in Lisbon region $(2.7 \%)$ and in central hospitals $(3.0 \%, p<0.001)$. For episodes of heart failure, we found a readmissions rate of $6.7 \%$.

Discussion and Conclusion: Most of the differences found are consistent with those described in other Western countries. Readmission episodes, whose rates have been increasing in Portugal, are associated with higher hospital fatality rates.

Keywords: Patient Readmission; Portugal; Quality of Health Care; Hospital Mortality.

\section{INTRODUÇÃO}

Uma gestão hospitalar eficiente deverá ter em conta as necessidades do doente, a organização e segurança hospitalar, e a sustentabilidade financeira do hospital. Actualmente, devido à crise económica registada nos países ocidentais, torna-se cada vez mais necessário aumentar a eficiência económica no sector hospitalar e reduzir o desperdício. Vários estudos evidenciaram que elevadas taxas de reinternamentos estão relacionadas com um aumento das despesas no sector da saúde, com o decréscimo da qualidade dos cuidados de saúde e com elevadas taxas de mortalidade hospitalar. ${ }^{1-4}$

Uma readmissão hospitalar é passível de ser definida como uma nova hospitalização ocorrida num período de 1, 2, 4 ou 12 meses após ter sido conferida alta, ${ }^{5}$ embora esta definição não seja consensual no seio da comunidade científica. ${ }^{4}$ De facto, em termos gerais, as taxas de reinter-

1. Departamento de Ciências da Informação e da Decisão em Saúde. Faculdade de Medicina. Universidade do Porto. Porto. Portugal.

2. Centro de Investigação em Tecnologias e Sistemas de Informação em Saúde. Faculdade de Medicina. Universidade do Porto (CINTESIS). Porto. Portugal.

Recebido: 22 de Março de 2013 - Aceite: 09 de Setembro de 2013 | Copyright @ Ordem dos Médicos 2013 
namentos nos EUA para indivíduos adultos variam entre $5 \%$ e $29 \%$, devendo-se essa diferença, em parte, à falta de uniformidade na definição de readmissão hospitalar. ${ }^{4} \mathrm{Na}$ Europa, as taxas de reinternamentos aparentam ser similares - em Espanha, Alonso et al. obtiveram uma taxa de readmissão hospitalar de $26 \%$ em $2001 .^{6}$

Nos EUA, a pressão económica tem levado a um decréscimo do tempo de internamento, algo que poderá levar a um aumento do número de reinternamentos hospitalares, ${ }^{4,7,8}$ o que acarreta consequências nefastas..$^{1-3}$ De facto, alguns reinternamentos poderiam ser evitados, na medida em que estão relacionadas com a atribuição precoce de alta, ou com a prestação de cuidados de saúde deficientes ou inadequados. ${ }^{5}$ De acordo com Benbassat e Taragin, entre $9 \%$ a $48 \%$ dos reinternamentos poderiam ser prevenidos, na medida em que estão associados a cuidados inadequados durante o período de hospitalização. ${ }^{5}$ Para além disso, de acordo com o mesmo estudo, entre $12 \%$ a $75 \%$ dos reinternamentos poderiam ser evitados com recurso à educação dos doentes, a uma correcta avaliação antes da decisão da alta, e à manutenção de cuidados no domicílio. ${ }^{5} \mathrm{Na}$ verdade, a manutenção de cuidados domiciliários a longo-prazo aparenta desempenhar um papel deveras importante, no que concerne à prevenção de reinternamentos hospitalares..$^{9,10}$

Apesar de os reinternamentos serem frequentemente utilizados como um indicador de qualidade de saúde, vários estudos demonstraram que estes dificilmente poderão avaliar este parâmetro. ${ }^{11,12} \mathrm{~A}$ título de exemplo, Luthi et al (2004) estudaram a ocorrência de reinternamentos hospitalares em doentes com insuficiência cardíaca e concluíram que os primeiros não constituíam um indicador válido de qualidade dos cuidados hospitalares. ${ }^{13} \mathrm{Um}$ outro estudo, conduzido por Kossovsky et al (2000), verificou que, no caso de doentes com insuficiência cardíaca congestiva, os reinternamentos hospitalares não-planeados estariam mais relacionadas com as características clínicas e demográficas do que com a qualidade dos cuidados de saúde no hospital. ${ }^{14}$

De facto, vários estudos concluíram que certas características dos doentes, tais como a idade, o género, a história médica e as comorbilidades apresentadas, se relacionam com a taxa de reinternamentos, ${ }^{1,3-5,12,15-19}$ que também varia em função de certas características hospitalares. ${ }^{7,20} A$ título de exemplo, está descrito que as taxas de reinternamentos são mais elevadas em hospitais centrais, de maiores dimensões (com mais de 200 camas), mais complexos, com tecnologias mais sofisticadas e mais especializados, na medida em que estes recebem uma maior proporção de doentes com problemas mais graves. ${ }^{20}$

No que se refere às características dos doentes, tanto nos EUA como na Europa, as taxas de reinternamentos são maiores nos indivíduos mais velhos. ${ }^{3-5,16}$ Já relativamente ao género, aparentemente, verificam-se maiores taxas de reinternamentos nos indivíduos do sexo masculino. ${ }^{3-5,16}$ Por fim, quanto às doenças mais associadas com os reinternamentos hospitalares, destacam-se as do aparelho cardio- vascular e respiratório. ${ }^{5}$

A redução das taxas de reinternamentos hospitalares permite reduzir os custos hospitalares e melhorar a qualidade dos tratamentos, ${ }^{5,21}$ e, consequentemente, a caracterização dos reinternamentos hospitalares assume grande importância. Não obstante, foi realizado um reduzido número de estudos com o intuito de analisar os reinternamentos hospitalares portugueses. Uma vez que os reinternamentos hospitalares em Portugal ainda se encontram pouco estudados, reduzi-los torna-se um objectivo mais difícil de atingir, sendo este o principal motivo que nos levou a investigar este tema.

Com este trabalho, pretende-se estudar a dimensão actual dos reinternamentos hospitalares portugueses não-planeados ocorridos num período de 30 dias após alta, bem como a sua evolução ao longo da última década. Por outro lado, este trabalho tem por objectivo estudar as taxas de mortalidade hospitalar associadas aos reinternamentos hospitalares. Pretende-se ainda analisar a variação das taxas de readmissão hospitalar em função das regiões portuguesas, do contexto hospitalar e de características dos doentes (nomeadamente a Grande Categoria Diagnóstica atribuída, o género e a idade).

Para além de estudar os reinternamentos hospitalares numa perspectiva mais geral, este trabalho procura ainda descrever, em particular, os reinternamentos em doentes com insuficiência cardíaca em função das características demográficas e do contexto hospitalar. A escolha da insuficiência cardíaca como patologia de estudo deve-se, não só ao seu elevado impacto socio-económico, ${ }^{22-24}$ mas também ao facto de já terem sido conduzidos estudos que evidenciam que a adopção de estratégias dirigidas a doentes com insuficiência cardíaca e elevado risco de readmissões permite reduzir o risco de readmissões hospitalares. ${ }^{25,26}$

Por fim, neste trabalho procura-se ainda comparar os valores encontrados com resultados de estudos idênticos levados a cabo noutros países Europeus e da América do Norte.

\section{MATERIAL E MÉTODOS \\ Análise Estatística}

Analisámos uma base de dados fornecida pela Autoridade Central para os Sistemas de Saúde (ACSS), que contém um registo de todas as hospitalizações ocorridas em hospitais públicos portugueses (com excepção dos episódios ocorridos nos arquipélagos dos Açores e da Madeira) num período de nove anos (altas desde 2000 até 2008). Foram considerados somente os episódios de internamento, de tal modo que os episódios de ambulatório foram excluídos desta análise.

Esta base de dados contém ainda informação demográfica e clínica relativa aos doentes que estiveram hospitalizados, bem como informação relativa ao hospital onde cada internamento ocorreu, algo que nos permitiu estudar as taxas de reinternamentos hospitalares de acordo com diferentes variáveis. Todavia, uma vez que nesta base de dados o código de identificação associado a cada doente é 
diferente de ano para ano e consoante o hospital, foi apenas possível estudar os reinternamentos intra-hospitalares ocorridos num período de 30 dias após alta, entre Fevereiro e Dezembro de cada ano. Deste modo, foram apenas contabilizados os reinternamentos hospitalares não-planeados ocorridos num período de 30 dias após alta e cuja Grande Categoria Diagnóstica atribuída fosse igual à do primeiro internamento.

No que concerne às variáveis analisadas, procedeu-se ao estudo das taxas de reinternamentos em função do ano, com o objectivo de estudar a sua evolução - note-se que, como referido anteriormente, a base de dados analisada continha episódios ocorridos num período de nove anos (desde 2000 até 2008). Foi ainda calculada a taxa de mortalidade hospitalar nos episódios de reinternamento e nos de não-reinternamento.

Por outro lado, procurámos saber se existia uma correlação entre as taxas de reinternamentos hospitalares e o nível de complexidade hospitalar. Deste modo, para agrupar os hospitais públicos de acordo com a sua complexidade, utilizámos uma classificação do Sistema Nacional de Saúde, que divide estas instituições de saúde em Hospitais Centrais, Hospitais Distritais, e Hospitais Distritais Nível 1, sendo os Hospitais Centrais os mais complexos e os Hospitais Distritais Nível 1 os menos complexos. ${ }^{27}$ Já para estudar a variação das taxas de reinternamentos com a região geográfica, adoptámos a classificação NUTS II, que divide Portugal Continental em cinco regiões - Norte, Centro, Lisboa, Alentejo e Algarve. ${ }^{28}$

Calculámos ainda as taxas de reinternamentos associadas a cada Grande Categoria Diagnóstica, algo que permitiu saber quais os grupos de doenças e perturbações que apresentam maiores taxas de reinternamentos. De referir que esta classificação resulta do agrupamento dos episódios hospitalares em 26 áreas diagnósticas exclusivas (geralmente, dizendo a respeito a uma especialidade médica particular). ${ }^{29}$

Procedemos ainda à análise das taxas de reinternamentos hospitalares de acordo com o género e grupo etário dos indivíduos readmitidos. No que concerne à última variável, os indivíduos foram agrupados em cinco grupos etários (0-14 anos, 15-24 anos, 25-44 anos, 45-64 anos e +65 anos), sendo esta divisão frequentemente utilizada pelo Instituto Nacional de Estatística (INE). ${ }^{30}$

A análise estatística da base de dados foi conseguida com recurso aos testes do qui-quadrado, para variáveis discretas nominais, conjuntamente com o teste para averiguação de associação linear (linear-by-linear association test) para variáveis discretas ordinais, tendo sido considerados estatisticamente significativos valores de $p$ inferiores a 0,05. A análise de dados foi levada a cabo recorrendo ao software de tratamento estatístico de dados SPSS, versão 18.0 (SPSS, Inc., Chicago IL, EUA).

Procedimentos similares foram adoptados para calcular as taxas de readmissões hospitalares a 30 dias em episódios não-planeados de insuficiência cardíaca. Foram considerados como episódios de insuficiência cardíaca todos aqueles cujo diagnóstico principal ou secundário estava preenchido com um dos seguintes códigos da ICD-9-CM: 428.x, 402.01, 402.11, 402.91, 404.01, 404.03, 404.11, 404.13, 404.91 e 404.93 .

\section{Pesquisa de Artigos}

Os artigos utilizados para comparação de resultados foram obtidos após pesquisa em três bases de dados on-line - PubMed, ISI Web of Knowledge e Google Scholar. Após ter sido realizada uma pesquisa definida, os artigos foram seleccionados com base em critérios de inclusão e de exclusão - seriam incluídos artigos onde se verificasse a determinação de taxas de reinternamentos hospitalares não-planeados, ocorridos num período de um mês e pelo mesmo grupo diagnóstico; enquanto artigos que não

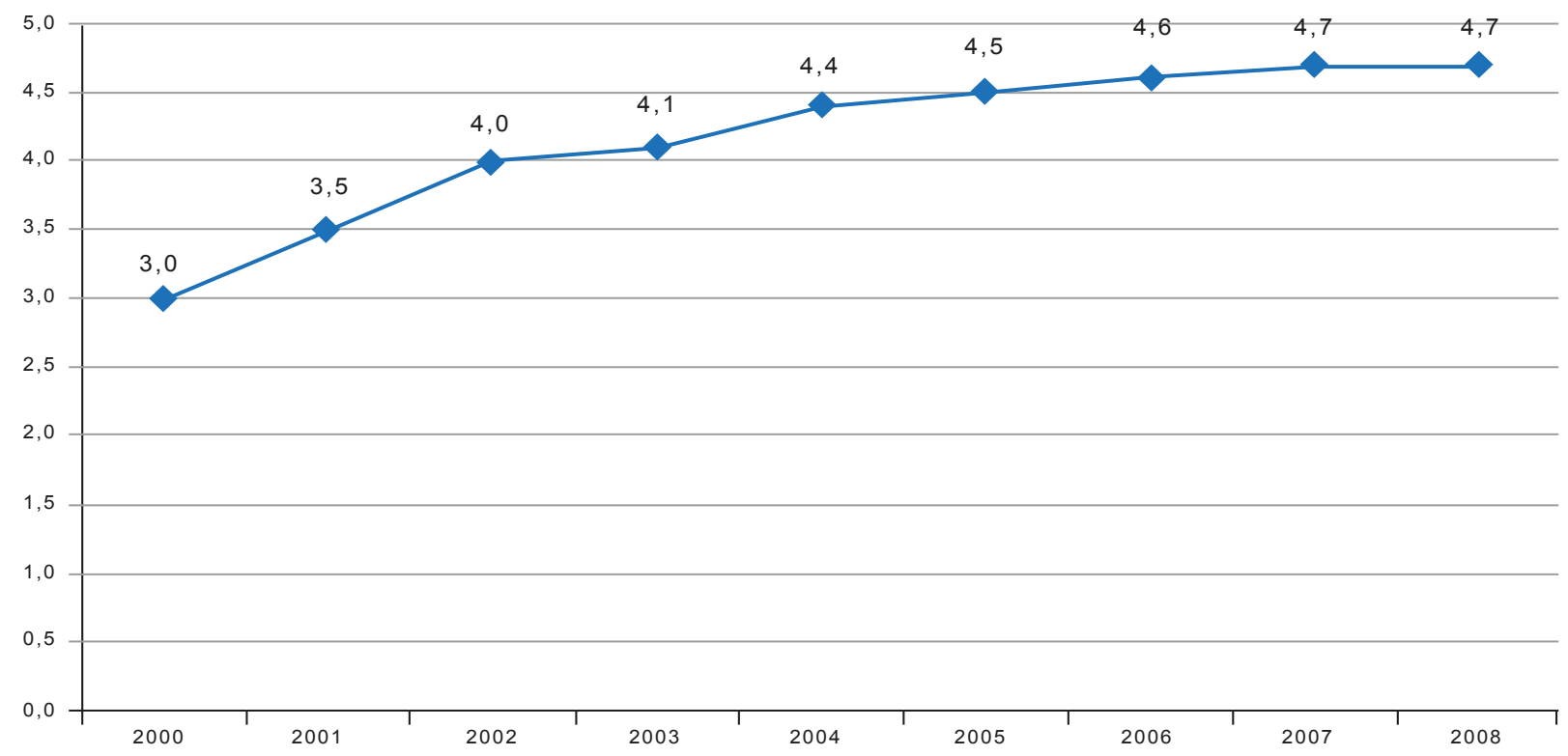

Figura 1 - Taxas de reinternamentos (não-planeados pela mesma GCD) por ano 
Reinternamentos

Reinternados

Não-reinternados

\section{Género*}

Masculino

Feminino

Faixa etária**

0-14 anos

15-24 anos

25-44 anos

45-64 anos

$\geq 65$ anos

Região*

Norte

Centro

Lisboa

Alentejo

Algarve

Tipo de Hospital ${ }^{* *}$

Hospitais Centrais

Hospitais Distritais

Hospitais Distritais Nível 1

Grande categoria diagnóstica (Doenças e Perturbações)*

0 - Pré-Grandes Categorias Diagnósticas

1 - Sistema Nervoso

2 - Olho

3 - Ouvido, nariz, boca e garganta

4 - Aparelho respiratório

5 - Aparelho circulatório

6 - Aparelho digestivo

7 - Sistema hepatobiliar e pâncreas

8 - Sistema músculo-esquelético e tecido conjuntivo

9 - Pele, tecido celular subcutâneo e mama

10 - Doenças e perturbações endócrinas nutricionais e metabólicas

11 - Rim e aparelho urinário

12 - Aparelho genital masculino

13 - Aparelho genital feminino

14 - Gravidez, parto e puerpério

15 - Recém-nascidos e lactentes com afecções do período peri natal

16 - Sangue/órgãos hematopoiéticos e doenças imunológicas

17 - Doenças e perturbações mieloproliferativas e mal diferenciadas

18 - Doenças infecciosas e parasitárias

19 - Doenças e perturbações mentais

20 - Uso de álcool/droga e perturbações mentais orgânicas induzidas por álcool ou droga

21 - Traumatismos, intoxicações e efeitos tóxicos de drogas

22 - Queimaduras

23 - Factores com influência no estado de saúde e outros contactos com os serviços de saúde

24 - Infecções pelo vírus da imunodeficiência humana

25 - Traumatismos múltiplos significativos

${ }^{*}$ Diferenças estatisticamente significativas, de acordo com o teste do qui-quadrado $(p<0,001)$

** Diferenças estatisticamente significativas de acordo com o teste de associação linear (linear-by-linear association) $(p<0,001)$
4,5 
estivessem escritos em português, inglês, espanhol, ou italiano, seriam eliminados.

\section{RESULTADOS}

Considerando todos os episódios de internamento hospitalar ( $n=8001277$ ), foi encontrada uma taxa total de reinternamentos de $6,8 \%$. Por outro lado, considerando apenas os episódios hospitalares não-programados $(n=5$ 514 331), foi encontrada uma taxa de reinternamentos hospitalares a 30 dias de $6,4 \%$. Se considerarmos os reinternamentos no período de um mês em hospitalizações não-planeadas, mas de acordo com a mesma GCD, obtemos uma taxa de 4,1\% ( $n=227459)$. De referir que, todos os dados subsequentemente apresentados são relativos apenas aos reinternamentos não-planeados pela mesma GCD.

A análise das taxas de reinternamentos por ano permite constatar que o valor desta taxa aumentou significativamente entre 2000 e 2008 ( $p<0,001)$, tendo esse aumento sido mais acentuado entre 2000 e 2004 . O ano de 2008 foi o único no qual não ocorreu um aumento da taxa de readmissões face ao ano anterior (Fig. 1).

No que diz respeito à mortalidade, os dados obtidos corroboram a ideia de que os reinternamentos hospitalares estão associados a maiores taxas de mortalidade hospitalar. Deste modo, 9,5\% dos episódios de reinternamentos não-planeados resultaram na morte do utente, contra apenas $5,6 \%$ dos restantes episódios não-planeados $(p<0,001)$.

No que concerne ao estudo das taxas de reinternamentos por GCD, foram também encontradas diferenças estatisticamente significativas, tendo a CGD 17 (que englobava os diagnósticos referentes às doenças e perturbações mieloproliferativas e mal diferenciadas) sido a que apresentou a maior taxa de reinternamentos. Todavia, entre os episódios de reinternamento, a taxa de mortalidade intra-hospitalar é superior para a CGD 4, que diz respeito a patologias do aparelho respiratório (Tabela 1).

Em termos demográficos, a taxa de reinternamentos registada nos indivíduos do sexo masculino é superior à dos indivíduos do sexo feminino (4,5 vs. 3,9\%), sendo esta diferença estatisticamente significativa $(p<0,001)$. Para além disso, a taxa de mortalidade intra-hospitalar nos reinternamentos em homens é muito superior à dos reinternamentos em mulheres (12,1 vs. 7,1, Tabela 1$)$.

Também foram encontradas diferenças estatisticamente significativas $(p<0,001)$ relativamente à variação das

Tabela 2 - Percentagem de hospitalizações não-planeadas e reinternamentos de acordo com a faixa etária para cada grau de complexidade do hospital

\begin{tabular}{|c|c|c|c|c|c|c|c|c|c|c|c|}
\hline & & \multicolumn{2}{|c|}{$0-14$ anos } & \multicolumn{2}{|c|}{ 15-24 anos } & \multicolumn{2}{|c|}{$25-44$ anos } & \multicolumn{2}{|c|}{ 45-64 anos } & \multicolumn{2}{|c|}{$\geq 65$ anos } \\
\hline & & $\mathrm{H}^{*}$ & $\mathrm{R}^{* *}$ & $\mathrm{H}^{*}$ & $\mathrm{R}^{* *}$ & $\mathrm{H}^{*}$ & $\mathrm{R}^{* *}$ & $\mathrm{H}^{*}$ & $\mathrm{R}^{* *}$ & $\mathrm{H}^{*}$ & $\mathrm{R}^{* *}$ \\
\hline & Hospitais Centrais & 21,8 & 2,2 & 7,1 & 2,8 & 23,4 & 2,6 & 15,6 & 3,6 & 32,1 & 3,5 \\
\hline \multirow{2}{*}{$\begin{array}{l}\text { Complexidade } \\
\text { do Hospital }\end{array}$} & Hospitais Distritais & 23,8 & 2,7 & 7,3 & 4,3 & 21,7 & 4,0 & 13,4 & 6,0 & 33,7 & 6,1 \\
\hline & $\begin{array}{c}\text { Hospitais Distritais } \\
\text { Nível } 1\end{array}$ & 16,5 & 4,6 & 5,6 & 4,4 & 15,1 & 4,8 & 14,3 & 6,4 & 48,6 & 6,7 \\
\hline
\end{tabular}

\footnotetext{
${ }^{*}$ Hospitalizações $(\%)$

** Taxa de reinternamentos (\%)
}

Tabela 3 - Percentagem de hospitalizações e reinternamentos de acordo com o tipo de hospital em cada região de Portugal Continental

\begin{tabular}{|c|c|c|c|c|c|c|c|}
\hline & & \multicolumn{2}{|c|}{$\begin{array}{l}\text { Hospitais } \\
\text { Centrais }\end{array}$} & \multicolumn{2}{|c|}{$\begin{array}{l}\text { Hospitais } \\
\text { Distritais }\end{array}$} & \multicolumn{2}{|c|}{$\begin{array}{c}\text { Hospitais } \\
\text { Distritais Nível } 1\end{array}$} \\
\hline & & $\mathrm{H}^{*}$ & $\mathrm{R}^{* *}$ & $\mathrm{H}^{*}$ & $\mathrm{R}^{* *}$ & $\mathrm{H}^{*}$ & $\mathrm{R}^{* *}$ \\
\hline \multirow{5}{*}{ Região } & Norte & 35,7 & 3,5 & 55,4 & 5,7 & 9,0 & 5,6 \\
\hline & Centro & 25,6 & 2,2 & 68,3 & 5,0 & 6,2 & 6,4 \\
\hline & Lisboa & 58,6 & 2,9 & 40,7 & 2,3 & 0,7 & 5,2 \\
\hline & Alentejo & 0,0 & - & 95,9 & 5,0 & 4,1 & 5,0 \\
\hline & Algarve & 0,0 & - & 100,0 & 4,9 & 0,0 & - \\
\hline
\end{tabular}

\footnotetext{
${ }^{*}$ Hospitalizações(\%)

** Taxa de reinternamentos (\%)
} 
taxas de reinternamentos por faixa etária, observando-se que estas taxas (tal como as taxas de mortalidade intra-hospitalar associadas) aumentam a par com a faixa etária (de $2,6 \%$ na faixa dos 0 aos 14 anos, até $5,3 \%$ na faixa dos maiores ou iguais a 65 anos).

No que diz respeito à região de localização do hospital, também foram encontradas diferenças estatisticamente significativas $(p<0,001)$, sendo Lisboa a região com a menor taxa de reinternamentos $(2,7 \%)$. Já no extremo oposto, encontra-se o Alentejo (5,1\%). Contudo, em termos de mortalidade intra-hospitalar associada aos reinternamentos, esta é menor no Norte $(7,9 \%)$ e maior no Alentejo $(11,7 \%)$ e em Lisboa (11,4\%) (Tabela 1).

Por outro lado, a percentagem de reinternamentos parece variar inversamente com a complexidade do hospital (Tabela 1), de tal modo que os Hospitais Centrais são aqueles que apresentam uma menor percentagem de reinternamentos $(3,0 \%$ vs. $4,7 \%$ e $5,9 \%$ nos restantes dois grupos, $p<0,001)$. Esta relação poderá ser parcialmente explicada pelo facto de a percentagem de hospitalizações de utentes com idade igual ou superior a 65 anos também variar em relação inversa com o grau de complexidade do hospital (Tabela 2). No entanto, mesmo por faixa etária, esta percentagem de reinternamentos é sempre consideravelmente menor nos hospitais centrais face aos restantes grupos. Por sua vez, a relação entre a complexidade hospitalar e a taxa de reinternamentos poderá parcialmente explicar o facto de as taxas de reinternamentos variarem de acordo com a região do país. De facto, Lisboa é simultaneamente a região com menor taxa de reinternamentos e com maior taxa de utentes atendidos em Hospitais Centrais (Tabela 3). Por oposição, o Alentejo e o Algarve, caracterizados pelas suas elevadas taxas de reinternamentos, são regiões desprovidas de Hospitais Centrais (Tabela 3 ).

\section{Reinternamentos Hospitalares em Doentes com Insufi- ciência Cardíaca}

Considerando todos os episódios não-programados de internamento por insuficiência cardíaca ( $n=315321)$, foi encontrada uma taxa de reinternamentos hospitalares a 30 dias de 6,7\% ( $n=21$ 165). Entre 2000 e 2008, o valor da taxa de reinternamentos em doentes com insuficiência cardíaca aumentou significativamente $(p<0,001)$. Embora tenha sido alvo de flutuações mais amplas, no cômputo geral, a mortalidade hospitalar aumentou no período descrito tanto nos indivíduos reinternados como nos indivíduos não-reinternados, tendo esse aumento sido superior nos indivíduos reinternados (Tabela 4 ).

De qualquer modo, não se registaram diferenças significativas na mortalidade hospitalar registada entre os doentes com insuficiência cardíaca reinternados $(17,2 \%)$ e não-reinternados $(16,9 \%)(p=0,367)$. De facto, contrariamente ao que seria expectável, no Alentejo, a mortalidade hospitalar é significativamente superior entre os doentes não-reinternados $(20,6 \%$ vs. $18,7 \%, p=0,047)$, registando-se um quadro similar entre os indivíduos com 0-14 anos $(15,1 \%$ vs. $6,0 \%, p=0,03)$ (Tabela 4$)$.
Tal como expectável, a taxa de reinternamentos registada nos indivíduos do sexo masculino é significativamente superior à dos indivíduos do sexo feminino ( 7,1 vs. $6,4 \%$, $p<0,001)$. Para além disso, foram encontradas diferenças estatisticamente significativas $(p<0,001)$ relativamente à variação das taxas de reinternamentos por faixa etária, sendo estas superiores na faixa etária dos 0 aos14 anos $(11,3 \%)$ e assumindo valores similares nas restantes faixas etárias. Por oposição, a mortalidade intra-hospitalar é superior em indivíduos de idade mais avançada (Tabela 4).

No que concerne à região, também se registam diferenças estatisticamente significativas (Tabela 4). De facto, nos doentes com insuficiência cardíaca, a taxa de reinternamentos é inferior na região de Lisboa $(4,7 \%)$ e superior no Algarve $(8,6 \%)$ e Alentejo $(8,1 \%)$. Estas duas últimas regiões também se destacam como sendo aquelas com maior mortalidade intra-hospitalar em doentes reinternados (20,2\% e 18,7\%, respectivamente). Por oposição, o Norte constitui a região onde a mortalidade intra-hospitalar em doentes reinternados atinge valores mais baixos (16,4\%).

No que diz respeito à complexidade hospitalar, os Hospitais Centrais são aqueles que apresentam uma menor percentagem de reinternamentos $(4,5 \%)$, por comparação com os Hospitais Distritais e Distritais Nível 1 (7,7\% e 8,3\%, respectivamente). Por outro lado, os Hospitais Distritais são aqueles que apresentam maior mortalidade hospitalar em doentes com insuficiência cardíaca (Tabela 4).

\section{Comparação com Outros Estudos}

Não foi encontrado nenhum estudo de âmbito nacional com o qual fosse possível a comparação de resultados. Todavia, foram encontrados vários artigos onde foram estudadas as taxas de reinternamentos não-planeados a um mês pelo mesmo grupo de diagnóstico num determinado hospital ou numa determinada cidade (Tabela 5).

\section{DISCUSSÃO}

Os resultados obtidos mostram que a taxa de reinternamentos hospitalares aumentou no período estudado, algo que é consistente com a literatura. , $^{4,8}$

As taxas de mortalidade hospitalar são superiores em indivíduos readmitidos, comparativamente aos indivíduos não-readmitidos (algo consistente com o que é descrito na literatura), ${ }^{3,4}$ o que provavelmente é explicado pelo facto de os reinternamentos hospitalares serem mais frequentes em casos de maior gravidade, bem como por uma possível ineficácia da prestação de cuidados, aquando do primeiro internamento.

De modo consistente com estudos anteriores, foram obtidas taxas de reinternamento superiores para indivíduos do sexo masculino. Isso poder-se-á dever ao facto de os homens apresentarem maior mortalidade e maior severidade, quando comparados a mulheres da mesma idade. ${ }^{16}$

Tal como expectável, a taxa de reinternamentos é significativamente maior em indivíduos mais idosos, provavelmente devido ao facto de a sua saúde ser mais frágil e de apresentarem mais comorbilidades. Na verdade, em doen- 
Tabela 4 - Taxas de reinternamentos não-planeados em doentes com insuficiência cardíaca, em função do ano, género, faixa etária, região do país e tipo de hospital.

\begin{tabular}{|c|c|c|c|}
\hline & \multirow{2}{*}{$\begin{array}{c}(\%) \\
\text { Reinternamentos }\end{array}$} & \multicolumn{2}{|c|}{ Mortalidade (\%) } \\
\hline & & Reinternados & Não-reinternados \\
\hline \multicolumn{4}{|l|}{ Ano $^{* *}$} \\
\hline 2000 & 4,7 & 15,6 & 16,4 \\
\hline 2001 & 5,4 & 16,4 & 16,4 \\
\hline 2002 & 6,5 & 17,2 & 17,2 \\
\hline 2003 & 6,5 & 18,4 & 17,3 \\
\hline 2004 & 6,7 & 18,0 & $16,2^{\dagger}$ \\
\hline 2005 & 6,8 & 16,8 & 17,3 \\
\hline 2006 & 7,2 & 17,2 & 16,9 \\
\hline 2007 & 7,9 & 16,9 & 17,1 \\
\hline 2008 & 7,8 & 17,3 & 17,3 \\
\hline \multicolumn{4}{|l|}{ Género* } \\
\hline Masculino & 7,1 & 18,2 & 17,1 \\
\hline Feminino & 6,4 & 16,2 & 16,7 \\
\hline \multicolumn{4}{|l|}{ Faixa etária** } \\
\hline $0-14$ anos & 11,3 & 6,0 & $15,1^{\dagger}$ \\
\hline 15-24 anos & 6,5 & 3,3 & 12,6 \\
\hline $25-44$ anos & 6,8 & 9,5 & 9,8 \\
\hline $45-64$ anos & 6,9 & 10,6 & $9,3^{+}$ \\
\hline$\geq 65$ anos & 6,7 & 18,2 & 18,0 \\
\hline \multicolumn{4}{|l|}{ Região* } \\
\hline Norte & 7,4 & 16,4 & 15,3 \\
\hline Centro & 7,0 & 17,1 & 17,1 \\
\hline Lisboa & 4,7 & 17,4 & 17,4 \\
\hline Alentejo & 8,1 & 18,7 & $20,6^{\dagger}$ \\
\hline Algarve & 8,6 & 20,2 & 20,1 \\
\hline \multicolumn{4}{|l|}{ Tipo de Hospital ${ }^{* *}$} \\
\hline Hospitais Centrais & 4,5 & 16,1 & 16,2 \\
\hline Hospitais Distritais & 7,7 & 17,7 & 17,6 \\
\hline Hospitais Distritais Nível 1 & 8,3 & 16,2 & 14,8 \\
\hline
\end{tabular}

* Diferenças estatisticamente significativas encontradas para os valores apresentados na coluna "Reinternamentos (\%)", de acordo com o teste do qui-quadrado ( $p<0,001)$

** Diferenças estatisticamente significativas encontradas para os valores apresentados na coluna "Reinternamentos(\%)", de acordo com o teste de associação linear (linear-by-linear association) $(p<0,001)$

† Diferenças estatisticamente significativas entre a mortalidade nos reinternados e não-reinternados. Os valores $p$ obtidos foram de 0,$024 ; 0,03 ; 0,039$ e 0,047 ; respectivamente para as diferenças da mortalidade em 2004, na faixa etária 0-14 anos, na faixa etária 45-64 anos e no Alentejo.

tes mais velhos, a morbilidade e a incapacidade funcional são considerados factores de risco para os reinternamentos hospitalares. ${ }^{31}$

Contrariamente ao que seria esperado, a complexidade do hospital varia inversamente com a taxa de readmissões. Isto poder-se-á dever ao facto de os hospitais mais complexos apresentarem melhor desempenho e eficiência. Por outro lado, de acordo com os dados utilizados neste estudo, os Hospitais Distritais Nível 1 são aqueles que apresentam maior percentagem de utentes com idade igual ou superior a 65 anos (Tabela 2), que constitui a faixa etária com maior taxa de readmissões.

No que concerne à análise regional, os dados obtidos evidenciam que as taxas de reinternamentos são menores em Lisboa, algo que é passível de ser explicado pelo facto de os utentes daquela região serem maioritariamente aten- didos em Hospitais Centrais (onde a taxa de reinternamentos é menor). De facto, de acordo com os dados patentes na Tabela 3, 58,6\% das hospitalizações ocorridas em Lisboa são relativas a Hospitais Centrais (Lisboa é a região com maior percentagem de internamentos ocorridos neste tipo de hospitais). Por oposição, o Alentejo e o Algarve são as únicas regiões de Portugal Continental desprovidas de Hospitais Centrais, ${ }^{27}$ o que em parte poderá explicar o facto de terem uma taxa de reinternamentos superior.

Por outro lado, as variações regionais das taxas de reinternamentos poderão ainda ser parcialmente explicadas por diferenças etárias. De facto, a média de idades em Portugal Continental (que corresponde a 39,2 anos) é inferior às médias registadas no Alentejo e no Algarve $(42,6 \mathrm{e}$ 40,8 anos, respectivamente). ${ }^{30}$ Para além disso, a percentagem de indivíduos com idade igual ou maior a 65 anos é 
Tabela 5 - Taxas de reinternamentos não-planeados a um mês pelo mesmo grupo diagnóstico, obtidas em estudos realizados por autores estrangeiros

Estudo

População estudada

Doentes de um hospital de Montreal (Canadá) entre 1995-1996

Doentes de um hospital universitário de São Francisco (Califórnia, EUA) entre 2006 e 2008

Allaudeen et al, $2011^{43}$

García Ortega et al, $1998^{44}$

Maurer et al $2004^{45}$

Seoane González et al $2010^{46}$

van Walraven et al, $2010^{47}$
Doentes de um hospital de Algeciras (Espanha) entre 1995 e 1996

Todos os doentes do Departamento de Medicina Interna

de um hospital de Winterhur (Suíça) internados em Maio de 1998

Doentes de um hospital universitário de A Corunha (Espanha)

Doentes de onze hospitais de Ontário (Canadá) entre 2002 e 2006
Reinternamentos (\%)
9,6

4,4

3,5

4,1

8,4 *

7,3

* Este valor diz respeito à taxa de reinternamentos a 28 dias, e não a 30 dias.

superior no Alentejo e no Algarve, comparativamente à média nacional. De facto, em Portugal Continental, $16,5 \%$ dos indivíduos apresentam uma idade igual ou superior a 65 anos de idade. Já no Alentejo e no Algarve, esse indicador atinge os $22,3 \%$ e $18,6 \%$, respectivamente. ${ }^{30}$ De ressalvar que, apesar de o Centro se encontrar mais envelhecido que o Algarve (no Centro, a média de idades ronda os 40,79 anos e a proporção de indivíduos com idade igual ou superior a 65 anos ronda os 19,4\%), ${ }^{30}$ a primeira região dispõe de um vasto conjunto de Hospitais Centrais, que não existem no Algarve. ${ }^{27}$

Uma vez que não foi encontrado nenhum estudo de âmbito nacional onde fosse determinada a taxa de reinternamentos hospitalares não-planeados no período de um mês, pela mesma GCD, não é possível estabelecer uma comparação entre as taxas de reinternamentos de diferentes países. Todavia, não parece existir uma tendência geográfica para as taxas de reinternamentos, na medida em que os valores encontrados para os hospitais da América do Norte são similares aos encontrados para os hospitais Europeus.

Nos doentes com insuficiência cardíaca, os valores obtidos neste estudo revelaram-se inferiores aos obtidos na maioria dos estudos estrangeiros consultados - nos EUA, diferentes estudos sugerem que, nos doentes com insuficiência cardíaca, a taxa de reinternamentos por esta condição atinja valores superiores a $10 \%,{ }^{32}$ enquanto a taxa de reinternamentos por todas as causas atinja valores superiores a $20 \% .^{23,32,33}$ Os valores obtidos em estudos europeus parecem assemelhar-se aos norte-americanos. ${ }^{34,35}$ Não obstante, Allen et al obtiveram valores similares aos presentes neste artigo. ${ }^{36} \mathrm{~A}$ associação entre o sexo masculino e o maior risco de reinternamentos em doentes com insuficiência cardíaca encontra-se corroborada por outros estudos. ${ }^{37,38}$ Contudo, contrariamente ao que foi descrito neste estudo, a maior parte dos estudos encontrou uma relação directa entre a complexidade hospitalar e a taxa de reinternamentos. ${ }^{37,38}$

A principal limitação deste trabalho prende-se com o facto de apenas terem sido analisados episódios ocorridos em hospitais públicos e em Portugal Continental (o que exclui os internamentos registados em hospitais privados e nas Regiões Autónomas dos Açores e da Madeira). No entanto, estes episódios correspondem a cerca de $85 \%$ dos internamentos hospitalares em Portugal. Por outro lado, uma vez que, nos dados analisados, o código de identificação de cada doente muda de ano para ano e é diferente consoante o hospital, o valor obtido de percentagem de reinternamentos hospitalares poderá ser ligeiramente inferior ao valor real.

Também o facto de os dados analisados serem de natureza administrativa poderá constituir uma limitação à generalização dos resultados obtidos, na medida em que os dados podem revelar falta de precisão ou poderão não estar completos. ${ }^{39-41}$ Todavia, o uso de dados administrativos em estudos relacionados com a saúde é frequente, na medida em que estes dados são facilmente acessíveis e permitem uma análise relativamente barata. ${ }^{34}$

De notar ainda que, tanto quanto seja do nosso conhecimento, não existem artigos publicados nos quais sejam estudados os reinternamentos hospitalares não-planeados a 30 dias em Portugal, o que nos impede a comparação directa de resultados. Contudo, o facto de este ser o primeiro estudo do género em Portugal evidencia a utilidade dos resultados presentes neste artigo. $\mathrm{Na}$ verdade, os resultados apresentados neste artigo podem ser úteis num contexto de gestão hospitalar, dado que evidenciam quais os factores que estão mais associados a taxas mais elevadas de reinternamentos hospitalares. Isto poderá ter efeitos positivos no Sistema Nacional de Saúde, uma vez que a caracterização dos reinternamentos hospitalares deverá ser o 
ponto de partida para a sua redução. Para além disso, foram analisados todos os episódios registados em hospitais públicos de doentes agudos de Portugal Continental entre 2000 e 2008, o que permite tirar conclusões mais precisas e analisar a evolução das taxas de reinternamentos hospitalares ao longo de quase uma década.

\section{CONCLUSÕES}

De entre os factores associados a maiores taxas de reinternamento, destaque para os episódios ocorridos em doentes mais velhos, do sexo masculino, residentes fora de Lisboa e atendidos em hospitais com menor complexidade. A taxa de reinternamentos hospitalares sofreu um aumento nesta última década - ora, tendo em conta que as taxas de mortalidade hospitalar são superiores nos episódios de

\section{REFERÊNCIAS}

1. Jweinat JJ. Hospital readmissions under the spotlight. J Healthc Manag. 2010;55:252-64

2. Chrusch CA, Olafson KP, McMillan PM, Roberts DE, Gray PR. High occupancy increases the risk of early death or readmission after transfer from intensive care. Crit Care Med. 2009;37:2753-8.

3. Sicras MA, Navarro AR. Valoracion del reingreso hospitalario en el servicio de medicina interna. Med Clin. 1993;101:732-5.

4. Hasan M. Readmission of patients to hospital: still ill defined and poorly understood. Int J Qual Health Care. 2001;13:177-9.

5. Benbassat J, Taragin M. Hospital readmissions as a measure of quality of health care: advantages and limitations. Arch Intern Med. 2000;160:1074-81.

6. Alonso MJL, Llorente DB, Echegaray AM, Urbieta EMA, Gonzalez AC. Reingreso hospitalario en Medicina Interna. An Med Interna. 2001;18:248-54.

7. Heggestad T. Do hospital length of stay and staffing ratio affect elderly patients' risk of readmission? A nation-wide study of Norwegian hospitals. Health Serv Res. 2002;37:647-65.

8. Bueno H, Ross JS, Wang Y, Chen J, Vidan MT, Normand SL, et al. Trends in length of stay and short-term outcomes among Medicare patients hospitalized for heart failure, 1993-2006. JAMA. 2010;303:2141-7.

9. Damiani G, Federico B, Venditti A, Sicuro L, Rinaldi S, Cirio F, et al. Hospital discharge planning and continuity of care for aged people in an Italian local health unit: does the care-home model reduce hospital readmission and mortality rates? BMC Health Serv Res. 2009;9:22.

10. Vavouranakis I, Lambrogiannakis E, Markakis G, Dermitzakis A, Haroniti $Z$, Ninidaki $C$, et al. Effect of home-based intervention on hospital readmission and quality of life in middle-aged patients with severe congestive heart failure: a 12-month follow up study. Eur $\mathrm{J}$ Cardiovasc Nurs. 2003;2:105-11.

11. Rosenberg AL, Watts $C$. Patients readmitted to ICUs*: a systematic review of risk factors and outcomes. Chest. 2000;118:492-502.

12. Fernandez GJ, Martinez GM, Garcia RJ, Bueno CA, Lardelli CP, Garcia MM. Factores asociados a la incidencia de reingresos hospitalarios. Med Clin. 1997;108:4-8.

13. Luthi JC, Burnand B, McClellan WM, Pitts SR, Flanders WD. Is readmission to hospital an indicator of poor process of care for patients with heart failure? Qual Saf Health Care. 2004;13:46-51.

14. Kossovsky MP, Sarasin FP, Perneger TV, Chopard P, Sigaud P, Gaspoz J. Unplanned readmissions of patients with congestive heart failure: do they reflect in-hospital quality of care or patient characteristics? Am J Med. 2000;109:386-90.

15. Billings J, Dixon J, Mijanovich T, Wennberg D. Case finding for patients at risk of readmission to hospital: development of algorithm to identify high risk patients. BMJ. 2006;333:327.

16. Moloney ED, Bennett K, Silke B. Patient and disease profile of emergency medical readmissions to an Irish teaching hospital. Postgrad Med J. 2004;80:470-4.

17. Chin MH, Goldman L. Correlates of early hospital readmission or death in patients with congestive heart failure. Am J Cardiol. 1997;79:1640-4.

18. Anderson GF, Steinberg EP. Predicting hospital readmissions in the Medicare population. Inquiry. 1985;22:251-8. reinternamento, é fundamental proceder à adopção de medidas que visem diminuir as taxas de reinternamento.

\section{AGRADECIMENTOS}

Os autores gostariam de agradecer à Administração Central do Sistema de Saúde, IP (ACSS) pela disponibilização dos dados utilizados neste trabalho de investigação.

\section{CONFLITO DE INTERESSES}

Os autores declaram que não houve conflito de interesses na realização deste trabalho.

\section{FONTES DE FINANCIAMENTO}

Os autores declaram a inexistência de fontes de financiamento externas.

19. Romansky JB, Lyons JS, Lehner RK, West CM. Factors related to psychiatric hospital readmission among children and adolescents in state custody. Psychiatr Serv. 2003;54:356-62.

20. Ansari MZ, Collopy BT, Booth JL. Hospital characteristics associated with unplanned readmissions. Aust Health Rev. 1995;18:63-75.

21. Jha AK, Orav EJ, Epstein AM. Public reporting of discharge planning and rates of readmissions. N Engl J Med. 2009;361:2637-45.

22. Shah KB, Rahim S, Boxer RS. Heart failure readmissions. Curr Treat Options Cardiovasc Med. 2013;15:437-49.

23. Zaya M, Phan A, Schwarz ER. Predictors of re-hospitalization in patients with chronic heart failure. World J Cardiol. 2012;4:23-30.

24. Fida N, Pina IL. Trends in heart failure hospitalizations. Curr Heart Fail Rep. 2012;9:346-53

25. Basoor A, Doshi NC, Cotant JF, Saleh T, Todorov M, Choksi N, et al. Decreased readmissions and improved quality of care with the use of an inexpensive checklist in heart failure. Congest Heart Fail. 2013;19:200-6.

26. Amarasingham R, Patel PC, Toto K, Nelson LL, Swanson TS, Moore $\mathrm{BJ}$, et al. Allocating scarce resources in real-time to reduce heart failure readmissions: a prospective, controlled study. BMJ Qual Saf. 2013.

27. Ministério da Saúde. Diário da República, Portaria n. ${ }^{\circ} 281 / 2005$ de 17 de Março. [consultado em 2012 Jun 27]. Disponível em: http://www.dre. pt/pdf1s/2005/03/054B00/24302431.pdf.

28. Ministério da Saúde. Diário da República, Portaria n. ${ }^{\circ} 255 / 2002$ de 5 de Novembro. [consultado em 2012 Jun 27]. Disponível em http://dre.pt/pd f1sdip/2002/11/255A00/71017103.pdf.

29. Ministério da Saúde. Diário da República, Portaria n. ${ }^{\circ} 113 / 2006$ de 12 de Junho. [consultado em 2012 Jun 27]. Disponível em http://www.acss. min-saude.pt/Portals/0/Port_567_2006_12\%20de\%20Junho.pdf.

30. Instituto Nacional de Estatística. Resultados Definitivos dos Censos 2001 [consultado em 2012 Jun 27]. Disponível em: http://censos.ine. pt/xportal/xmain?xpid=CENSOS\&xpgid=ine_censos_publicacoes\&pce nsospagenumber=2.

31. Garcia-Perez L, Linertova R, Lorenzo-Riera A, Vazquez-Diaz JR, DuqueGonzalez B, Sarria-Santamera A. Risk factors for hospital readmissions in elderly patients: a systematic review. QJM. 2011;104:639-51.

32. Robertson J, McElduff P, Pearson SA, Henry DA, Inder KJ, Attia JR. The health services burden of heart failure: an analysis using linked population health data-sets. BMC Health Serv Res. 2012;12:103.

33. Jencks SF, Williams MV, Coleman EA. Rehospitalizations among patients in the Medicare fee-for-service program. $\mathrm{N}$ Engl $\mathrm{J}$ Med. 2009;360:1418-28.

34. Zapatero A, Barba R, Gonzalez N, Losa JE, Plaza S, Canora J, et al. Influence of obesity and malnutrition on acute heart failure. Rev Esp Cardiol. 2012;65:421-6.

35. Schiotz M, Price M, Frolich A, Sogaard J, Kristensen JK, Krasnik A, et al. Something is amiss in Denmark: a comparison of preventable hospitalisations and readmissions for chronic medical conditions in the Danish Healthcare system and Kaiser Permanente. BMC Health Serv Res. 2011;11:347.

36. Allen LA, Smoyer Tomic KE, Smith DM, Wilson KL, Agodoa I. Rates and predictors of 30-day readmission among commercially insured and Medicaid-enrolled patients hospitalized with systolic heart failure. Circ 
Heart Fail. 2012;5:672-9.

37. Coffey RM, Misra A, Barrett M, Andrews RM, Mutter R, Moy E. Congestive heart failure: who is likely to be readmitted? Med Care Res Rev. 2012;69:602-16.

38. Caminal J, Hermosilla E, Sanchez E, Urrea M, Puig P, Tomas L. Estudio longitudinal de los reingresos en pacientes con insuficiencia cardiaca en Cataluna, 1996-1999. Rev Esp Salud Publica. 2004;78:267-76.

39. Freitas A, Silva-Costa T, Lopes F, Garcia-Lema I, Teixeira-Pinto A, Brazdil $P$, et al. Factors influencing hospital high length of stay outliers. BMC Health Serv Res. 2012;12:265.

40. Freitas JA, Silva-Costa T, Marques B, Costa-Pereira A. Implications of Data Quality Problems within Hospital Administrative Databases. IFMBE Proceedings. 2010;29:3.

41. Torchiana DF, Meyer GS. Use of administrative data for clinical quality measurement. J Thorac Cardiovasc Surg. 2005;129:1223-5.

42. Abenhaim HA, Kahn SR, Raffoul J, Becker MR. Program description: a hospitalist-run, medical short-stay unit in a teaching hospital. CMAJ. 2000;163:1477-80.

43. Allaudeen N, Vidyarthi A, Maselli J, Auerbach A. Redefining readmission risk factors for general medicine patients. J Hosp Med. 2011;6:54-60.

44. Garcia Ortega C, Almenara Barrios J, Garcia Ortega JJ. Tasa de reingresos de un hospital comarcal. Rev Esp Salud Publica. 1998;72:10310.

45. Maurer PP, Ballmer PE. Hospital readmissions--are they predictable and avoidable? Swiss Med Wkly. 2004;134:606-11.

46. Seoane Gonzalez B, Nicolas Miguel R, Ramos Polledo V, Pellice Vazquez C, de la Iglesia Martinez F. Unidad de corta estancia medica. Causas de reingreso. Rev Clin Esp. 2010;210:118-23.

47. van Walraven C, Dhalla IA, Bell C, Etchells E, Stiell IG, Zarnke K, et al. Derivation and validation of an index to predict early death or unplanned readmission after discharge from hospital to the community. CMAJ. 2010;182:551-7. 
Bernardo SOUSA-PINTO, Ana Rita GOMES, Andreia OLIVEIRA, Carlos IVO, Gustavo COSTA, João RAMOS, Joel SILVA, Maria Carolina CARNEIRO, Maria João DOMINGUES, Maria João CUNHA,

Altamiro da COSTA-PEREIRA, Alberto FREITAS

\section{Reinternamentos Hospitalares em Portugal na Última Década \\ Acta Med Port 2013:26:711-720}

Publicado pela Acta Médica Portuguesa, a Revista Científica da Ordem dos Médicos

Av. Almirante Gago Coutinho, 151

1749-084 Lisboa, Portugal.

Tel: +351218428 215

E-mail: submissao@actamedicaportuguesa.com

www.actamedicaportuguesa.com

ISSN:0870-399X | e-ISSN: 1646-0758

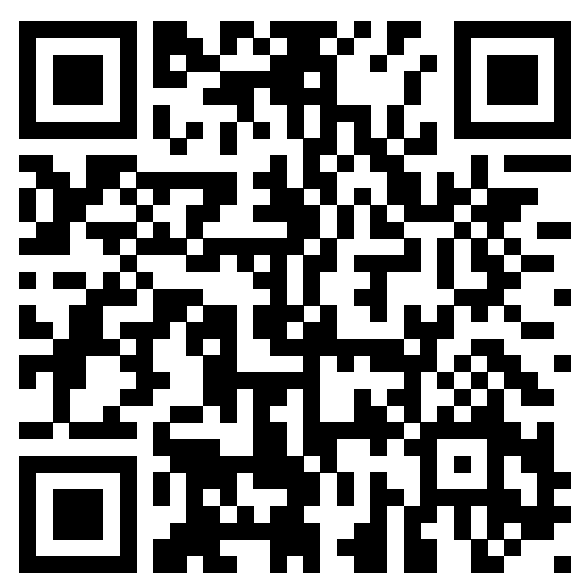

